\title{
Pulmonary neuroendocrine tumors: study of 266 cases focusing on clinicopathological characteristics, immunophenotype, and prognosis
}

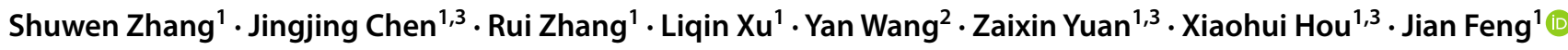

Received: 18 November 2021 / Accepted: 22 February 2022 / Published online: 6 March 2022

(c) The Author(s) 2022

\begin{abstract}
Objective Pulmonary neuroendocrine tumors (PNETs) consist of small-cell lung cancer (SCLC), large-cell neuroendocrine carcinoma (LCNEC), typical carcinoid (TC), and atypical carcinoid (AC). We aimed to analyze the immunophenotypic, metastatic, and prognostic risk factors for PNETs.

Materials and methods A total of 266 patients with PNETs were enrolled, including 219 patients with SCLC, 18 patients with LCNEC, 11 patients with TC, and 18 patients with AC. Clinicopathological characteristics and immunophenotypes were compared among the subtypes of PNETs. Risk factors for metastasis, progression-free survival (PFS), and overall survival (OS) were analyzed.

Results Thyroid transcription factor-1 (TTF-1) and the Ki-67 index were significantly different among subtypes of PNETs (all $P<0.05)$. Smoking (OR, 2.633; $P=0.031$ ), high pretreatment carcinoembryonic antigen (CEA $>5 \mathrm{ng} / \mathrm{ml}$ : OR, 3.084; $P=0.014)$, and poorly differentiated pathotypes $(P=0.001)$ were independent risk factors for lymph-node metastasis. Smoking (OR, 2.071; $P=0.027)$ and high pretreatment CEA $(\mathrm{OR}, 2.260 ; P=0.007)$ were independent risk factors for distant metastasis. Results of the multivariate Cox regression model showed pretreatment CEA (HR, 1.674; $P=0.008)$ and lymphocyte-monocyte ratio (LMR) $(\mathrm{HR}=0.478, P=0.007)$ were significantly associated with PFS; BMI $(P=0.031)$, lymphnode metastasis $(\mathrm{HR}=4.534, P=0.001)$, poorly differentiated pathotypes $(P=0.015)$, platelet-lymphocyte ratio (PLR) $(\mathrm{HR}=2.305, P=0.004)$, and LMR (HR $=0.524, P=0.045)$ were significantly associated with OS.

Conclusions PNETs are a group of highly heterogeneous tumors with different clinical manifestations, pathological features, and prognoses. Knowing clinicopathological characteristics and immunophenotypes of PNETs is significant for diagnosis. Pretreatment PLR, LMR, and CEA have certain value in the prognosis of PNETs.
\end{abstract}

Keywords Clinicopathological characteristics $\cdot$ Platelet-lymphocyte ratio $\cdot$ Lymphocyte-monocyte ratio · Carcinoembryonic antigen $\cdot$ Overall survival $\cdot$ Pulmonary neuroendocrine tumor

\section{Abbreviations}

PNET Pulmonary neuroendocrine tumor

SCLC Small cell lung cancer

Shuwen Zhang and Jingjing Chen have contributed equally to the work and are joint first authors.

Jian Feng

jfeng68@126.com

1 Department of Respiratory and Critical Care Medicine, Affiliated Hospital of Nantong University, $20 \mathrm{Xi}-\mathrm{Si}$ Road, Nantong 226001, Jiangsu, China

2 Department of Pathology, Affiliated Hospital of Nantong University, Nantong, Jiangsu, China

3 Nantong University, Nantong, Jiangsu, China
LCNEC Large cell neuroendocrine carcinoma

TC

$\mathrm{AC}$

PFS

OS

CEA

PLR

NLR

LMR

OR

HR

95\% CIs Typical carcinoid

Atypical carcinoid

Progression-free survival

Overall survival

Carcinoembryonic antigen

Platelet-lymphocyte ratio

Neutrophil-lymphocyte ratio

Lymphocyte-monocyte ratio

Odd ratio

Hazard ratio

ROC curve Receiver-operating characteristic curve

AUC

TTF-1
Area under the ROC curve

Thyroid transcription factor-1 


$\begin{array}{ll}\text { Syn } & \text { Synaptophysin } \\ \text { CgA } & \text { Chromogranin A } \\ \text { CK18 } & \text { Cytokeratin 18 } \\ \text { CK5/6 } & \text { Cytokeratin 5/6 } \\ \text { WHO } & \text { World Health Organization } \\ \text { NE } & \text { Neuroendocrine } \\ \text { PD-1 } & \text { Programmed death 1 } \\ \text { PD-L1 } & \text { Programmed death ligand 1 } \\ \text { LCs } & \text { Lung carcinoids } \\ \text { SEER } & \text { Surveillance, Epidemiology, and End Results } \\ \text { NSCLC } & \text { Non-small-cell lung cancer } \\ \text { EGFR } & \text { Epidermal growth factor receptor }\end{array}$

\section{Introduction}

Pulmonary neuroendocrine tumors (PNETs) are a unique subtype of primary lung cancer. It has been reported that PNETs represented 20\% of all lung cancers (Gustafsson et al. 2008). According to the 2015 World Health Organization (WHO) classification of lung tumors, PNETs include small-cell lung cancer (SCLC), large-cell neuroendocrine carcinoma (LCNEC), typical carcinoid (TC), and atypical carcinoid (AC). TC and AC are well-differentiated PNETs, whereas LCNEC and SCLC are poorly differentiated PNETs (Travis et al. 2015).

Even though PNET is rare, the incidence and prevalence are increasing, and the prognosis (especially SCLC and LCNEC) is terrible (Dasari et al. 2017). Fortunately, people are currently paying more attention to this kind of disease, especially for epidemiology, immunohistochemical molecular characteristics, and diagnosis. Current diagnostic methods primarily depend on neuroendocrine morphology and immunohistochemistry, such as cellular morphology, nuclear divisions, and the Ki-67 index. The 2015 WHO classification of lung tumors suggested that synaptophysin (Syn), chromogranin $\mathrm{A}(\mathrm{CgA})$, and $\mathrm{CD} 56$ are recommended as neuroendocrine (NE) markers, and Syn and CgA were suggested as the first-hand choice (Travis et al. 2015). Rekhtman (2010) revealed a mean (range) Ki-67 labeling index of $1.5(0-2.3 \%)$ for typical carcinoid tumors, $7.7(0-17 \%)$ for atypical carcinoid tumors, and 64 (25-96\%) for SCLC. Marchevsky et al. (2018) pointed out that the best cut-off value was Ki-67 $<5 \%$, which had great significance for diagnosis and prognosis.

Current strategies for tumor treatment include chemotherapy, radiotherapy, immunotherapy, targeted therapy, and surgery. The therapeutic options vary in the subtypes of PNETs. SCLC is initially highly sensitive to chemotherapy and radiotherapy, but most patients usually relapse and acquire resistant disease. The indications and results of surgical resection for SCLC remain controversial, and only a minority of patients with SCLC qualify for surgical resection. Programmed death 1 (PD-1) and programmed death ligand 1 (PD-L1) immune checkpoint inhibitors show good clinical activity in SCLC treatment. Atezolizumab combined with etoposide/carboplatin is recommended as the first-line treatment of extensive SCLC (Dingemans et al. 2021). However, there is no effective targeted therapy for SCLC. The first-line treatment modalities of LCNEC are quite different. In early stages, surgical resection is the preferred treatment, and patients can benefit from adjuvant chemotherapy at all operable stages (IA-IIIA). In its advanced stages, experience from the Dutch PALGA network has suggested that platinum-gemcitabine/taxanes may perform better than traditional platinum-etoposide approaches (Derks et al. 2017, 2018). For lung carcinoids (LCs), surgery is the preferred treatment. Large retrospective studies have reported no benefit of adjuvant therapy in either TCs or ACs. Therefore, the authors do not recommend routine adjuvant therapy in LCs (Anderson Jr et al. 2017; Daddi et al. 2014; García-Yuste et al. 2007; Nussbaum et al. 2015; Steuer et al. 2015).

To analyze the prognosis of PNETs, most previous studies have focused on Western populations. These large population-based studies usually download cases of PNETs in some databases for analyses [such as Surveillance, Epidemiology, and End Results (SEER) database] (Dasari et al. 2017; Doll et al. 2018). Databases provide clinical diagnosis, treatment, and prognosis information of different histopathological cancers and can help us better understand the disease. However, even the same disease may have diverse characteristics in different countries and regions. Their sample size is large, but practical applicability is limited. There are also some studies collecting cases in real clinical practice, but the sample size is small (Kim et al. 2020; Yeh and Chou 2014). Prior studies have implied that gender, age at diagnosis, tumor diameter, metastasis, stage, and first-line treatment modalities are associated with the prognosis of PNETs (Yang et al. 2019; Yeh and Chou 2014). However, only a limited number of studies have analyzed the clinical detection and survival outcomes of lung neuroendocrine neoplasms. Yet, high pretreatment carcinoembryonic antigen (CEA), neutrophil-lymphocyte ratio (NLR), platelet-lymphocyte ratio (PLR), and low lymphocyte-monocyte ratio (LMR) are tightly associated with poor prognosis of non-small-cell lung cancer (NSCLC) (Chen et al. 2018; Grunnet and Sorensen 2012; Kuo et al. 2020). Thus, large-scaled studies based on Chinese population are urgently needed to explore the relationship between clinical tests and the prognosis of pulmonary neuroendocrine tumors.

In our study, we involved 266 lung neuroendocrine neoplasms patients who had received treatments at our 
institution over the last 8 years and analyzed the clinicopathological characteristics and immunohistochemical profile. Simultaneously, clinical test indices were included to generalize metastatic risk factors for PNETs and to identify the influence factors of progression-free survival (PFS) and overall survival (OS).

\section{Materials and methods}

\section{Materials}

We collected patients' clinical and pathological data from patients who were diagnosed with PNETs and given treatments at the Affiliated Hospital of Nantong University from January 2012 to December 2020. PNETs were diagnosed based on the 2015 WHO diagnostic criteria. These patients' chest imaging examination and pretreatment blood examination results are available. All of them received at least one treatment at our institution. The exclusion criteria included the following: combined with other primary tumors and/or acute infectious diseases and failure to follow-up.

\section{Methods}

The participants' general clinical and anthropometric information were obtained from medical records and recorded at baseline, such as name, age, gender, body mass index (BMI), smoking history, laterality, and tumor diameter. Cases were divided into SCLC, LCNEC, TC, and AC according to the grading and classification criteria of pulmonary neuroendocrine tumors of the 2015 WHO. For the stage of PNETs, the two-stage system was used, in which limited-stage PNETs are defined as disease confined to a single, tolerable radiation port (stage I-III), and extensive-stage PNETs are the diseases that has extended beyond a single tolerable port (stage IV). We retrospectively analyzed tumors diagnosis, stages, lymph-node metastasis, distant metastasis, immunohistochemical molecular characteristics, pretreatment CEA, NLR, PLR, LMR, and therapeutic measures. Syn, CgA, Cytokeratin 18 (CK18), TTF-1, Napsin A, Cytokeratin 5/6 (CK5/6), p63, and Ki-67 index were analyzed as a representative molecule for immunohistochemistry. Pretreatment blood sampling was performed to measure the neutrophil, lymphocyte, monocyte, platelet, and CEA levels. The NLR was determined as the neutrophil count divided by the lymphocyte count, PLR was determined as the platelet count divided by the lymphocyte count, and LMR was defined as the absolute lymphocyte count/the absolute monocyte count. PFS was calculated from the date of treatment initiation to the date of progression or death or last follow-up, and OS was defined as the time from the date of treatment initiation to the date of death or last follow-up. Follow-up was terminated on December 31, 2021. The above clinicopathological characteristics were grouped by whether there was metastasis, disease progression, and death separately, implying risk factors for metastasis and survival.

\section{Statistical analysis}

Clinicopathological characteristics and immunophenotype of SCLC, LCNEC, TC, and AC were compared using the Pearson's chi-squared test and Kruskal-Wallis $H$ test. Frequencies and percentages are reported for categorical variables, and medians with range or interquartile range are reported for continuous variables. Receiver-operating characteristic (ROC) curve analysis was performed to analyze the area under the ROC curve (AUC), and the Youden Index was used to identify the optimal cut-off values for NLR, PLR, and LMR. Univariate and multivariate logistic regression models were applied to identify risk factors for metastasis, and accurate estimate of odd ratios (ORs) and $95 \%$ confidence intervals (95\% CIs) were reported. To analyze the prognostic factors, univariate and multivariate Cox proportional hazard models were used, and the hazard ratios (HRs) and 95\% CIs were reported. Survival analysis was conducted and the significance of differences among groups was tested using the log-rank test. PFS and 5-year OS were investigated using the Kaplan-Meier method. Above analyses were performed using SPSS statistical package version 26.0 (SPSS, Chicago, IL, USA). $P$ value $<0.05$ was considered statistically significant. GraphPad Prism7 software (GraphPad Software, San Diego, CA, USA) was used to generate the ROC curves and survival curves.

\section{Results}

\section{The optimal cut-off point of PLR, NLR, and LMR}

To determine the potential prognostic role of NLR, PLR, and LMR in PNETs, ROC analysis was performed to identify the optimal cut-off point of these immune-inflammation indices. ROC curve analysis indicated an optimal cut-off PLR of 152.5 (AUC $=0.663,95 \% \mathrm{CI}=0.594-0.731$, sensitivity $=49.36 \%$, specificity $=82.56 \%$ ), an optimal cut-off NLR of $2.5(\mathrm{AUC}=0.654,95 \% \mathrm{CI}=0.584-0.725$, sensitivity $=64.74 \%$, specificity $=61.63 \%$ ), and optimal cut-off LMR of 2.9 (AUC of $0.668,95 \% \mathrm{CI}=0.598-0.738$, sensitivity $=76.74 \%$, specificity $=50.00 \%$ ) (Fig. 1). For each immune-inflammation index, patients were divided into two groups for further analysis $[$ PLR $\leq 152.5$ (low) and PLR $>152.5$ (high); NLR $\leq 2.5$ (low) and NLR $>2.5$ (high); LMR $\leq 2.9$ (low) and LMR $>2.9$ (high)]. 

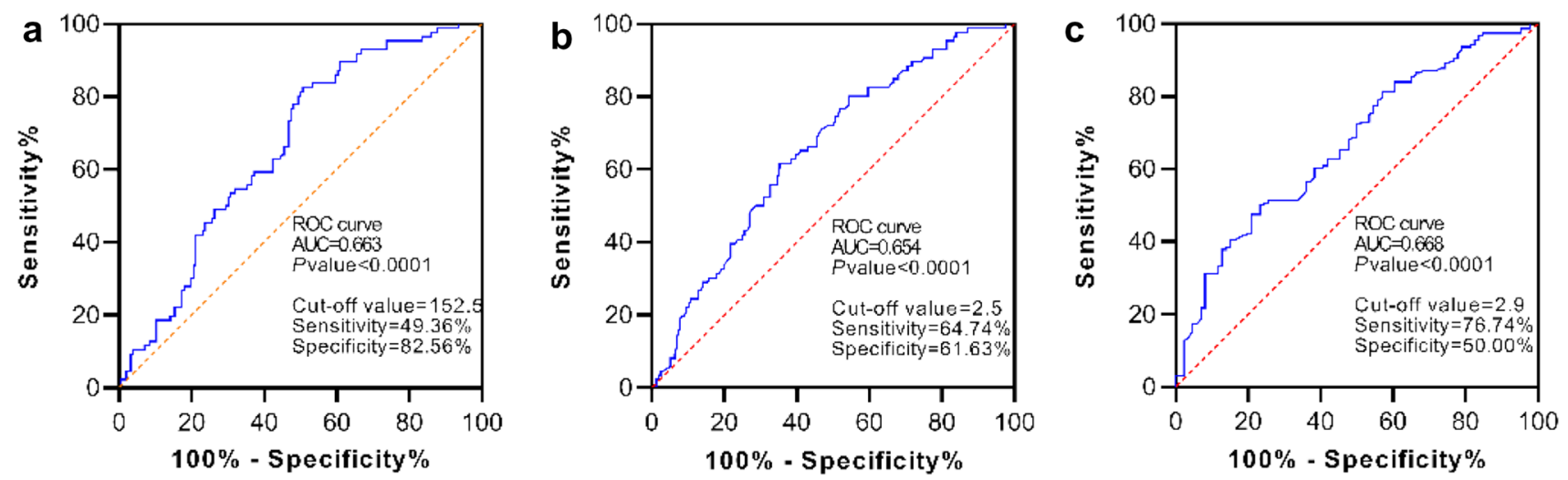

Fig. 1 ROC curve for immune-inflammation indices. a ROC curve for PLR; b ROC curve for NLR; $\mathbf{c}$ ROC curve for LMR. Abbreviations: ROC curve receiver-operating characteristic curve; PLR plate- let-lymphocyte ratio; NLR neutrophil-lymphocyte ratio; LMR lymphocyte-monocyte ratio

\section{Immunohistochemical molecular characteristics' comparison among PNETs}

To compare the differences in immunohistochemical profiles among PNETs, Syn, CgA, CK18, TTF-1, Napsin A, CK5/6, Ki-67, and p63 were examined (Table 2). SCLC had a higher positive rate of TTF-1 $(89.50 \%$ vs. $52.94 \%$ vs. $80.00 \%$ vs. $72.22 \%, P=0.000)$. The Ki-67 index was significantly higher in SCLC and LCNEC than in carcinoid tumors (the median: $80.00 \%$ vs. $75.00 \%$ vs. $2.00 \%$ vs $30.00 \%, P=0.000)$. Other immunohistochemical molecules (such as Syn, CgA, CK18, Napsin A, CK5/6, p63) did not show significant differences among the subtypes of PNETs.

\section{Metastatic risk factors' analysis of PNETs}

We employed univariate logistic regression analysis to explore the risk factors for metastasis. As shown in Table 3, gender (lymph-node metastasis: female, OR 0.322, 95\% CI 0.149-0.693, $P=0.004$; distant metastasis: female, OR $0.396,95 \%$ CI $0.173-0.905, P=0.028)$, age at diagnosis (lymph-node metastasis: $\geq 60$ years, OR $2.023,95 \% \mathrm{CI}$ 1.048-3.906, $P=0.036$; distant metastasis: $\geq 60$ years, OR $2.204,95 \%$ CI 1.216-3.995, $P=0.009$ ), smoking (lymphnode metastasis: OR 2.627, 95\% CI 1.367-5.050, $P=0.004$; distant metastasis: OR 1.962, 95\% CI 1.177-3.269, $P=0.010$ ), tumor size (taking tumor size $<3 \mathrm{~cm}$ as reference, lymph-node metastasis: 3-5 cm, OR 3.000, 95\% CI $1.399-6.434, P=0.005,5-7 \mathrm{~cm}$, OR $10.071,95 \%$ CI 2.297-44.168, $P=0.002$. Distant metastasis: $3-5 \mathrm{~cm}$, OR $1.880,95 \%$ CI $1.013-3.487, P=0.045,5-7 \mathrm{~cm}, \mathrm{OR}$ $2.965,95 \%$ CI $1.448-6.070, P=0.003 ;>7 \mathrm{~cm}$, OR 3.503 , 95\% CI 1.486-8.257, $P=0.004$ ), and pathotypes (taking SCLC as reference, lymph-node metastasis: LCNEC, OR 
Table 1 Clinical characteristics' comparison among PNETs

\begin{tabular}{|c|c|c|c|c|c|c|c|}
\hline & All & SCLC & LCNEC & $\mathrm{TC}$ & $\mathrm{AC}$ & $\chi^{2}$ & $P$ value \\
\hline Gender & & & & & & 16.157 & $0.001 *$ \\
\hline Male & $229(86.09)$ & $197(89.95)$ & $14(77.78)$ & $6(54.55)$ & $12(66.67)$ & & \\
\hline Female & $37(13.91)$ & $22(10.05)$ & $4(22.22)$ & $5(45.45)$ & $6(33.33)$ & & \\
\hline Age at diagnosis (years) & & & & & & 8.917 & $0.030 *$ \\
\hline$<60$ & $74(27.82)$ & $53(24.20)$ & $7(38.89)$ & $6(54.55)$ & $8(44.44)$ & & \\
\hline$\geq 60$ & $192(72.18)$ & $166(75.80)$ & $11(61.11)$ & $5(45.45)$ & $10(55.56)$ & & \\
\hline BMI $\left(\mathrm{kg} / \mathrm{m}^{2}\right)$ & & & & & & 1.963 & 0.925 \\
\hline$<18.5$ & $15(5.64)$ & $14(6.39)$ & $0(0.00)$ & $0(0.00)$ & $1(5.56)$ & & \\
\hline $18.5-23.9$ & $139(52.26)$ & $115(52.51)$ & $9(50.00)$ & $7(63.64)$ & $8(44.44)$ & & \\
\hline$>24$ & $112(42.11)$ & $90(41.10)$ & $9(50.00)$ & $4(36.36)$ & $9(50.00)$ & & \\
\hline Smoking & & & & & & 6.341 & 0.096 \\
\hline No & $118(44.36)$ & $92(42.01)$ & $7(38.89)$ & $8(72.73)$ & $11(61.11)$ & & \\
\hline Yes & $148(55.64)$ & $127(57.99)$ & $11(61.11)$ & $3(27.27)$ & $7(38.89)$ & & \\
\hline Tumor size $(\mathrm{cm})$ & & & & & & 15.069 & 0.058 \\
\hline$\leq 3$ & $100(37.59)$ & 74 (33.79) & $7(38.89)$ & $8(72.73)$ & $11(61.11)$ & & \\
\hline $3-5$ & $88(33.08)$ & 74 (33.79) & $9(50.00)$ & $1(9.09)$ & $4(22.22)$ & & \\
\hline $5-7$ & $49(18.42)$ & $44(20.09)$ & $2(11.11)$ & $2(18.18)$ & $1(5.56)$ & & \\
\hline$>7$ & $29(10.90)$ & $27(12.33)$ & $0(0.00)$ & $0(0.00)$ & $2(11.11)$ & & \\
\hline Laterality & & & & & & 5.437 & 0.410 \\
\hline Left & $107(40.23)$ & $92(42.01)$ & $7(38.89)$ & $5(45.45)$ & $3(16.67)$ & & \\
\hline Right & $148(55.64)$ & $117(53.42)$ & $11(61.11)$ & $6(54.55)$ & $14(77.78)$ & & \\
\hline Others & $11(4.14)$ & $10(4.57)$ & $0(0.00)$ & $0(0.00)$ & $1(5.56)$ & & \\
\hline Lymph-node metastasis & & & & & & 37.945 & $0.000 *$ \\
\hline No & 47 (17.67) & $24(10.96)$ & $5(27.78)$ & $7(63.64)$ & $11(61.11)$ & & \\
\hline Yes & $219(82.33)$ & $195(89.04)$ & $13(72.22)$ & $4(36.36)$ & $7(38.89)$ & & \\
\hline Distant metastasis & & & & & & 18.470 & $0.000^{*}$ \\
\hline No & $164(61.65)$ & $123(56.16)$ & $14(77.78)$ & $11(100.00)$ & $16(88.89)$ & & \\
\hline Yes & $102(38.35)$ & $96(43.84)$ & $4(22.22)$ & $0(0.00)$ & $2(11.11)$ & & \\
\hline Stage & & & & & & 10.553 & $0.012^{*}$ \\
\hline $\begin{array}{l}\text { Limited stage/stage } \\
\text { I-III }\end{array}$ & $154(57.89)$ & $117(53.42)$ & $13(72.22)$ & $9(81.82)$ & $15(83.33)$ & & \\
\hline \multirow{2}{*}{$\begin{array}{l}\text { Extensive stage/stage } \\
\text { IV }\end{array}$} & $112(42.11)$ & $102(46.58)$ & $5(27.78)$ & $2(18.18)$ & $3(16.67)$ & & \\
\hline & All & SCLC & LCNEC & $\mathrm{TC}$ & $\mathrm{AC}$ & $\chi^{2}$ & $P$ value \\
\hline CEA (ng/ml) & & & & & & 7.101 & 0.069 \\
\hline$\leq 5$ & $151(56.77)$ & $117(54.42)$ & $12(66.67)$ & $10(90.91)$ & $12(66.67)$ & & \\
\hline$>5$ & $111(41.73)$ & $98(45.58)$ & $6(33.33)$ & $1(9.09)$ & $6(33.33)$ & & \\
\hline PLR & & & & & & 6.988 & 0.066 \\
\hline$\leq 152.5$ & $157(61.09)$ & $121(57.35)$ & $14(82.35)$ & $8(72.73)$ & $14(77.78)$ & & \\
\hline$>152.5$ & $100(38.91)$ & $90(42.65)$ & $3(17.65)$ & $3(27.27)$ & $4(22.22)$ & & \\
\hline NLR & & & & & & 16.847 & $0.001 *$ \\
\hline$\leq 2.5$ & $105(40.86)$ & 77 (36.49) & $6(35.29)$ & $9(81.82)$ & $13(72.22)$ & & \\
\hline$>2.5$ & $152(59.14)$ & $134(63.51)$ & $11(64.71)$ & $2(18.18)$ & $5(27.78)$ & & \\
\hline LMR & & & & & & 5.576 & 0.130 \\
\hline$\leq 2.9$ & $104(40.47)$ & $92(43.60)$ & $6(35.29)$ & $2(18.18)$ & $4(22.22)$ & & \\
\hline$>2.9$ & $153(59.53)$ & $119(56.40)$ & $11(64.71)$ & $9(81.82)$ & $14(77.78)$ & & \\
\hline Treatment strategy & & & & & & 78.119 & $0.000^{*}$ \\
\hline Chemotherapy & $179(67.29)$ & $167(76.26)$ & $6(33.33)$ & $1(9.09)$ & $5(27.78)$ & & \\
\hline $\begin{array}{l}\text { Chemotherapy + radio- } \\
\text { therapy }\end{array}$ & $5(1.88)$ & $5(2.28)$ & $0(0.00)$ & $0(0.00)$ & $0(0.00)$ & & \\
\hline
\end{tabular}


Table 1 (continued)

\begin{tabular}{|c|c|c|c|c|c|c|c|}
\hline & All & SCLC & LCNEC & $\mathrm{TC}$ & $\mathrm{AC}$ & $\chi^{2}$ & $P$ value \\
\hline Surgery & $27(10.15)$ & $6(2.74)$ & $5(27.78)$ & $7(63.64)$ & $9(50.00)$ & & \\
\hline $\begin{array}{l}\text { Surgery + chemother- } \\
\text { apy/radiotherapy }\end{array}$ & $40(15.04)$ & $29(13.24)$ & $6(33.33)$ & $1(9.09)$ & $4(22.22)$ & & \\
\hline Others & $15(5.64)$ & $12(5.48)$ & $1(5.56)$ & $2(18.18)$ & $0(0.00)$ & & \\
\hline PFS & $\begin{array}{l}6.93 \\
\quad(3.77,13.45)\end{array}$ & $6.62(3.56,10.42)$ & $11.83(2.32,35.09)$ & $\begin{array}{l}52.86 \\
\quad(26.30,54.70)\end{array}$ & $17.39(5.74,41.93)$ & & $0.000^{*}$ \\
\hline OS & $\begin{array}{l}16.53 \\
(9.61,30.1)\end{array}$ & $14.97(9.37,26.48)$ & $21.42(4.98,44.40)$ & $\begin{array}{l}54.43 \\
\quad(32.70,82.00)\end{array}$ & $\begin{array}{l}32.02 \\
(17.58,41.93)\end{array}$ & & $0.000^{*}$ \\
\hline
\end{tabular}

PNETS pulmonary neuroendocrine tumors; SCLC small-cell lung cancer; $L C N E C$ large-cell neuroendocrine carcinoma; $A C$ atypical carcinoid; TC, typical carcinoid; $B M I$ body mass index; $C E A$ carcinoembryonic antigen; $P L R$ platelet-lymphocyte ratio; $N L R$ neutrophil-lymphocyte ratio; $L M R$ lymphocyte-monocyte ratio; $P F S$ progression-free survival; $O S$ overall survival

$* P<0.05$

Table 2 Immunohistochemical molecular characteristics comparison among PNETs

\begin{tabular}{|c|c|c|c|c|c|c|c|}
\hline & All & SCLC & LCNEC & $\mathrm{TC}$ & $\mathrm{AC}$ & $\chi^{2}$ & $P$ value \\
\hline Syn & & & & & & 6.767 & 0.052 \\
\hline Negative & $26(10.32)$ & 18 (8.74) & $5(27.78)$ & $2(18.18)$ & $1(5.88)$ & & \\
\hline Positive & 226 (89.68) & $188(91.26)$ & $13(72.22)$ & $9(81.82)$ & $16(94.12)$ & & \\
\hline $\mathrm{CgA}$ & & & & & & 5.875 & 0.112 \\
\hline Negative & $76(31.67)$ & $63(32.14)$ & $9(50.00)$ & $2(20.00)$ & $2(12.50)$ & & \\
\hline Positive & $164(68.33)$ & $133(67.86)$ & $9(50.00)$ & $8(80.00)$ & $14(87.50)$ & & \\
\hline CK18 & & & & & & 4.313 & 0.210 \\
\hline Negative & $6(2.93)$ & $4(2.35)$ & $1(6.25)$ & $1(12.50)$ & $0(0.00)$ & & \\
\hline Positive & 199 (97.07) & $166(97.65)$ & $15(93.75)$ & $7(87.50)$ & $11(100.00)$ & & \\
\hline TTF-1 & & & & & & 16.618 & $0.000^{*}$ \\
\hline Negative & $36(14.69)$ & $21(10.50)$ & $8(47.06)$ & $2(20.00)$ & $5(27.78)$ & & \\
\hline Positive & $209(85.31)$ & 179 (89.50) & $9(52.94)$ & $8(80.00)$ & $13(72.22)$ & & \\
\hline Napsin A & & & & & & 4.153 & 0.183 \\
\hline Negative & $221(97.36)$ & 177 (97.79) & $16(88.89)$ & $11(100.00)$ & $17(100.00)$ & & \\
\hline Positive & $6(2.64)$ & $4(2.21)$ & $2(11.11)$ & $0(0.00)$ & $0(0.00)$ & & \\
\hline CK5/6 & & & & & & 2.247 & 0.466 \\
\hline Negative & $193(95.54)$ & $151(95.57)$ & $16(88.89)$ & $9(100.00)$ & $17(100.00)$ & & \\
\hline Positive & $9(4.46)$ & $7(4.43)$ & $2(11.11)$ & $0(0.00)$ & $0(0.00)$ & & \\
\hline Ki67 (\%) & $80.00(60.00,80.00)$ & $80.00(70.00,86.25)$ & $75.00(52.50,80.00)$ & $2.00(1.00,2.75)$ & $30.00(20.00,50.00)$ & & $0.000^{*}$ \\
\hline P63 & & & & & & 3.041 & 0.339 \\
\hline Negative & $135(86.54)$ & $103(83.74)$ & $11(91.67)$ & $8(100.00)$ & $13(100.00)$ & & \\
\hline Positive & $21(13.46)$ & $20(16.26)$ & $1(8.33)$ & $0(0.00)$ & $0(0.00)$ & & \\
\hline
\end{tabular}

PNETs pulmonary neuroendocrine tumors; SCLC small-cell lung cancer; $L C N E C$ large-cell neuroendocrine carcinoma; $A C$ atypical carcinoid; $T C$ typical carcinoid; Syn synaptophysin; $C g A$ chromogranin A; CK18 Cytokeratin 18; TTF-1 thyroid transcription factor-1; CK5/6 Cytokeratin $5 / 6$

${ }^{*} P<0.05$

$0.320,95 \%$ CI $0.105-0.976, P=0.045$; TC, OR 0.070 , 95\% CI $0.019-0.258, P=0.000$; AC, OR $0.078,95 \%$ CI $0.028-0.221, P=0.000$. Distant metastasis: AC, OR 0.160, 95\% CI 0.036-0.713, $P=0.016$ ), pretreatment CEA (lymphnode metastasis: > 5 ng/ml, OR 3.678, 95\% CI 1.693-7.991,
$P=0.001$; distant metastasis: $>5 \mathrm{ng} / \mathrm{ml}$, OR $2.557,95 \%$ CI $1.531-4.272, P=0.000$ ), PLR (lymph-node metastasis: $>152.5$, OR $2.407,95 \%$ CI $1.162-4.988, P=0.018$; distant metastasis: $>152.5$, OR: $2.249,95 \%$ CI 1.337-3.782, $P=0.002$ ), NLR (lymph-node metastasis: $>2.5$, OR 2.053, 


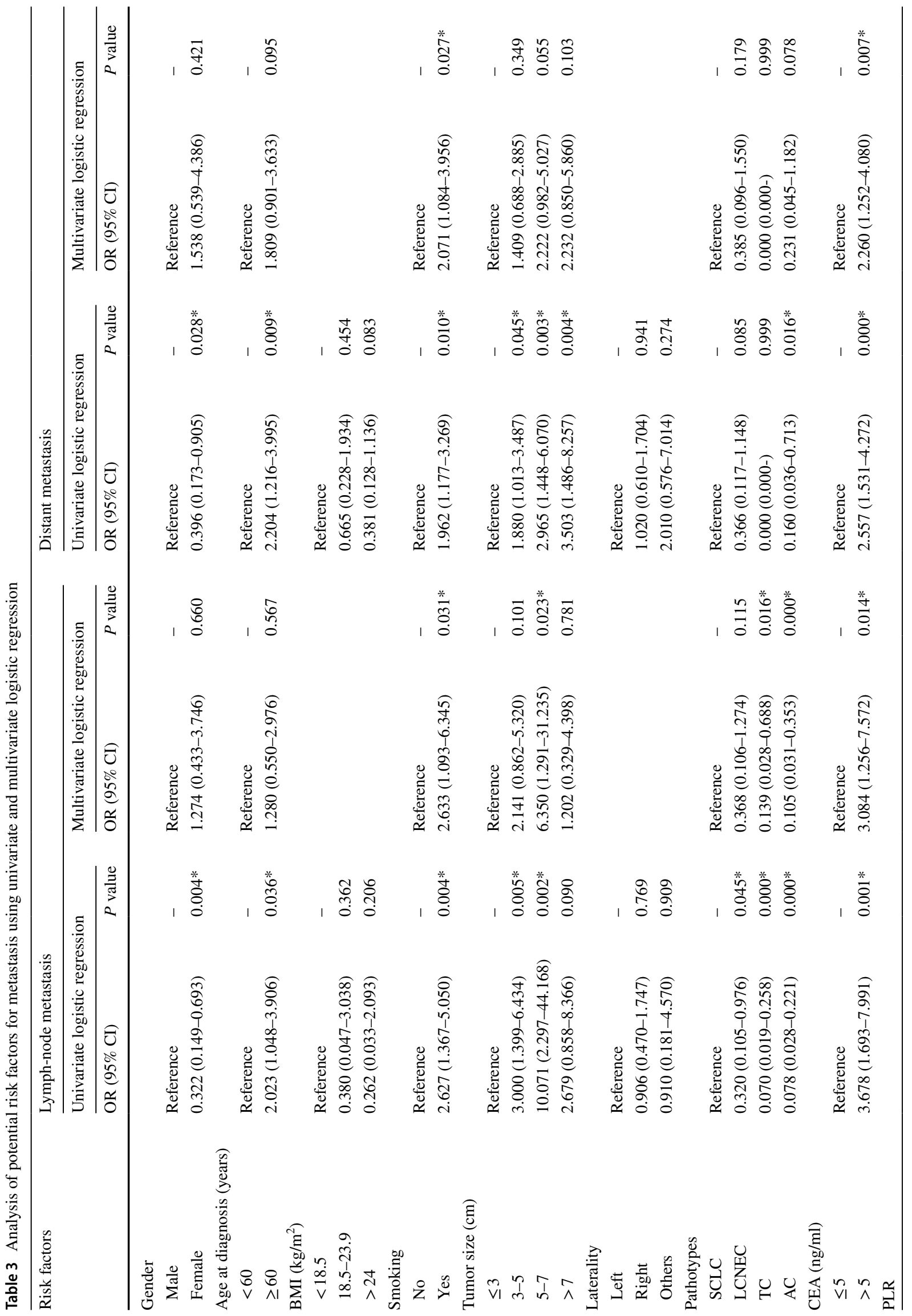


95\% CI 1.083-3.891, $P=0.027$; distant metastasis: $>2.5$, OR $2.402,95 \%$ CI 1.397-4.128, $P=0.002$ ), LMR (lymphnode metastasis: $>2.9$, OR $0.334,95 \%$ CI $0.158-0.706$, $P=0.004$; distant metastasis: $>2.9$, OR $0.350,95 \% \mathrm{CI}$ $0.208-0.591, P=0.000$ ) were significantly associated with metastasis. We further conducted multivariate logistic regression analysis to explore the independent risk factors for metastasis. Smoking (OR 2.633; 95\% CI 1.093-6.345; $P=0.031$ ), pathotypes (taking SCLC as reference, TC, OR $0.139,95 \%$ CI $0.028-0.688, P=0.016$; AC, OR $0.105,95 \%$ CI $0.031-0.353, P=0.000)$, and pretreatment CEA ( $>5 \mathrm{ng} /$ $\mathrm{ml}$, OR 3.084; 95\% CI 1.256-7.572; $P=0.014$ ) were proved to be the independent factors of lymph-node metastasis. Smoking (OR 2.071; 95\% CI 1.084-3.956; $P=0.027$ ) and pretreatment CEA ( $>5 \mathrm{ng} / \mathrm{ml}$, OR: $2.260 ; 95 \%$ CI $1.252-4.080 ; P=0.007)$ were the independent risk factors for distant metastasis (Table 3).

\section{Survival risk factors' analysis of PNETs}

At the end of the follow-up time, PFS was calculated in all 266 patients, but only 248 patients were calculated for OS, and 18 patients were lost to follow-up. A total of 227 (85.34\%) patients presented disease progression, and 161 (64.92\%) patients died of PNETs. Cox proportional hazards model was used to evaluate the potential predictors, as shown in Table 4. The univariate analysis revealed that PFS was significantly associated with gender (HR $0.553 ; 95 \%$ CI $0.360-0.849 ; P=0.007$ ), smoking (HR $1.360 ; 95 \%$ CI $1.042-1.774 ; P=0.024)$, tumor size ( $3-5 \mathrm{~cm}$ : HR, 1.863; 95\% CI $1.349-2.572 ; P=0.000 ; 5-7 \mathrm{~cm}$ : HR $2.634 ; 95 \%$ CI $1.808-3.838 ; P=0.000 ;>7 \mathrm{~cm}$ : HR $2.412 ; 95 \%$ CI 1.545-3.766; $P=0.000$ ), metastasis (lymph-node metastasis: HR 4,390; 95\% CI 2.788-6.912; $P=0.000$; distant metastasis: HR $2.824 ; 95 \%$ CI $2.148-3.713 ; P=0.000$ ), pathotypes (taking SCLC as reference, LCNEC: HR 0.406; 95\% CI 0.220-0.749; $P=0.004$; TC: HR 0.098; 95\% CI 0.031-0.310; $P=0.000$; AC: HR 0.221; 95\% CI $0.104-0.472 ; P=0.000$ ), Ki-67 index (HR 1.016; 95\% CI 1.009-1.024; $P=0.000$ ), pretreatment CEA ( $>5 \mathrm{ng} /$ ml: HR 2.105; 95\% CI 1.609-2.754; $P=0.000)$, PLR ( $>152.5$ : HR $1.827 ; 95 \%$ CI 1.391-2.399; $P=0.000$ ), NLR (> 2.5: HR 1.562; 95\% CI 1.185-2.058; $P=0.002)$, LMR (>2.9: HR 0.481; 95\% CI, 0.366-0.631; $P=0.000$ ) and first-line treatment modalities (surgery: HR $0.127 ; 95 \% \mathrm{CI}$ $0.064-0.252 ; P=0.000$; surgery combined with chemotherapy or radiotherapy: HR 0.338 ; $95 \%$ CI $0.227-0.506$; $P=0.000)$. OS was significantly associated with gender (female: HR 0.488 ; 95\% CI 0.290-0.820; $P=0.007$ ), age at diagnosis ( $\geq 60$ years: HR 1.464; 95\% CI 1.027-2.088; $P=0.035)$, BMI $\left(18.5-23.9 \mathrm{~kg} / \mathrm{m}^{2}: \mathrm{HR}, 0.476 ; 95 \% \mathrm{CI}\right.$ $0.265-0.856 ; P=0.013 ;>24 \mathrm{~kg} / \mathrm{m}^{2}:$ HR $0.413 ; 95 \% \mathrm{CI}$ $0.227-0.752 ; P=0.004)$, tumor size $(3-5 \mathrm{~cm}$ : HR 1.738; 
Table 4 Analysis of potential risk factors for PFS and OS using univariate and multivariate Cox proportional hazard models

\begin{tabular}{|c|c|c|c|c|c|c|c|c|}
\hline \multirow[t]{3}{*}{ Risk Factors } & \multicolumn{4}{|c|}{ PFS: cox regression analysis $(N=266,227$ progression events) } & \multicolumn{4}{|c|}{ OS: cox regression analysis ( $N=248,161$ dead events) } \\
\hline & \multicolumn{2}{|l|}{ Univariable analysis } & \multicolumn{2}{|l|}{ Multivariate analysis } & \multicolumn{2}{|l|}{ Univariable analysis } & \multicolumn{2}{|l|}{ Multivariate analysis } \\
\hline & $\mathrm{HR}(95 \% \mathrm{CI})$ & $P$ value & $\mathrm{HR}(95 \% \mathrm{CI})$ & $P$ value & HR $(95 \% \mathrm{CI})$ & $P$ value & $\mathrm{HR}(95 \% \mathrm{CI})$ & $P$ value \\
\hline \multicolumn{9}{|l|}{ Gender } \\
\hline Male & Reference & - & Reference & - & Reference & - & Reference & - \\
\hline Female & $0.553(0.360-0.849)$ & $0.007 *$ & $1.240(0.681-2.257)$ & 0.483 & $0.488(0.290-0.820)$ & $0.007 *$ & $1.073(0.522-2.202)$ & 0.849 \\
\hline \multicolumn{9}{|c|}{$\begin{array}{l}\text { Age at diagnosis } \\
\text { (years) }\end{array}$} \\
\hline$<60$ & Reference & - & & & Reference & - & Reference & - \\
\hline$\geq 60$ & $1.197(0.890-1.609)$ & 0.234 & & & $1.464(1.027-2.088)$ & $0.035^{*}$ & $0.583(0.337-1.010)$ & 0.054 \\
\hline \multicolumn{9}{|l|}{$\operatorname{BMI}\left(\mathrm{kg} / \mathrm{m}^{2}\right)$} \\
\hline$<18.5$ & Reference & - & & & Reference & - & Reference & - \\
\hline $18.5-23.9$ & $0.683(0.393-1.189)$ & 0.177 & & & $0.476(0.265-0.856)$ & $0.013 *$ & $0.249(0.088-0.701)$ & $0.009 *$ \\
\hline$>24$ & $0.554(0.315-0.974)$ & $0.040 *$ & & & $0.413(0.227-0.752)$ & $0.004 *$ & $0.262(0.085-0.804)$ & $0.019 *$ \\
\hline \multicolumn{9}{|l|}{ Smoking } \\
\hline No & Reference & - & Reference & - & Reference & - & & \\
\hline Yes & $1.360(1.042-1.774)$ & $0.024 *$ & $1.130(0.770-1.659)$ & 0.531 & $1.289(0.939-1.768)$ & 0.116 & & \\
\hline \multicolumn{9}{|l|}{ Laterality } \\
\hline Left & Reference & - & & & Reference & - & & \\
\hline Right & $0.991(0.755-1.301)$ & 0.948 & & & $0.955(0.691-1.320)$ & 0.781 & & \\
\hline Others & $1.817(0.966-3.415)$ & 0.064 & & & $1.735(0.890-3.384)$ & 0.106 & & \\
\hline \multicolumn{9}{|c|}{ Tumor size $(\mathrm{cm})$} \\
\hline$\leq 3$ & Reference & - & Reference & - & Reference & - & Reference & - \\
\hline $3-5$ & $1.863(1.349-2.572)$ & $0.000 *$ & $1.758(1.079-2.867)$ & $0.024^{*}$ & $1.738(1.196-2.526)$ & $0.004 *$ & $1.686(0.942-3.016)$ & 0.079 \\
\hline $5-7$ & $2.634(1.808-3.838)$ & $0.000 *$ & $1.600(0.950-2.693)$ & 0.077 & $1.750(1.115-2.747)$ & $0.015^{*}$ & $1.362(0.706-2.627)$ & 0.357 \\
\hline$>7$ & $2.412(1.545-3.766)$ & $0.000^{*}$ & $1.302(0.716-2.365)$ & 0.387 & $1.761(1.041-2.980)$ & $0.035^{*}$ & $1.099(0.513-2.354)$ & 0.809 \\
\hline \multicolumn{9}{|c|}{$\begin{array}{l}\text { Lymph-node metas- } \\
\text { tasis }\end{array}$} \\
\hline No & Reference & - & Reference & - & Reference & - & Reference & - \\
\hline Yes & $4.390(2.788-6.912)$ & $0.000 *$ & $1.748(0.939-3.256)$ & 0.078 & $5.633(2.963-10.707)$ & $0.000 *$ & $4.534(1.887-10.894)$ & $0.001 *$ \\
\hline \multicolumn{9}{|c|}{ Distant metastasis } \\
\hline No & Reference & - & Reference & - & Reference & - & Reference & - \\
\hline Yes & $2.824(2.148-3.713)$ & $0.000 *$ & $1.115(0.751-1.655)$ & 0.590 & $2.761(2.015-3.782)$ & $0.000 *$ & $1.043(0.637-1.706)$ & 0.868 \\
\hline \multicolumn{9}{|l|}{ Pathotypes } \\
\hline SCLC & Reference & - & Reference & - & Reference & - & Reference & - \\
\hline LCNEC & $0.406(0.220-0.749)$ & $0.004 *$ & $1.420(0.632-3.191)$ & 0.396 & $0.598(0.313-1.144)$ & 0.120 & $3.154(1.182-8.419)$ & $0.022 *$ \\
\hline TC & $0.098(0.031-0.310)$ & $0.000^{*}$ & $0.123(0.023-0.664)$ & $0.015^{*}$ & $0.170(0.054-0.538)$ & $0.003 *$ & $0.187(0.036-0.962)$ & $0.045^{*}$ \\
\hline $\mathrm{AC}$ & $0.221(0.104-0.472)$ & $0.000 *$ & $0.408(0.141-1.178)$ & 0.097 & $0.354(0.165-0.759)$ & $0.008 *$ & $0.773(0.237-2.523)$ & 0.669 \\
\hline \multirow[t]{3}{*}{ Risk Factors } & \multicolumn{4}{|c|}{ PFS: cox regression analysis $(N=266,227$ progression events) } & \multicolumn{4}{|c|}{ OS: cox regression analysis $(N=248,161$ dead events) } \\
\hline & \multicolumn{2}{|l|}{ Univariable analysis } & Multivariate analysis & & Univariable analysis & & Multivariate analysis & \\
\hline & $\operatorname{HR}(95 \% \mathrm{CI})$ & $P$ value & $\operatorname{HR}(95 \% \mathrm{CI})$ & $P$ value & $\mathrm{HR}(95 \% \mathrm{CI})$ & $P$ value & $\mathrm{HR}(95 \% \mathrm{CI})$ & $P$ value \\
\hline CEA (ng/ml) & & & & & & & & \\
\hline$\leq 5$ & Reference & - & Reference & - & Reference & - & Reference & - \\
\hline$>5$ & $2.105(1.609-2.754)$ & $0.000 *$ & $1.674(1.141-2.456)$ & $0.008^{*}$ & $2.147(1.563-2.947)$ & $0.000 *$ & $1.223(0.782-1.913)$ & 0.378 \\
\hline PLR & & & & & & & & \\
\hline$\leq 152.5$ & Reference & - & Reference & - & Reference & - & Reference & - \\
\hline$>152.5$ & $1.827(1.391-2.399)$ & $0.000 *$ & $1.374(0.842-2.244)$ & 0.204 & $2.502(1.820-3.441)$ & $0.000 *$ & $2.305(1.311-4.055)$ & $0.004^{*}$ \\
\hline NLR & & & & & & & & \\
\hline$\leq 2.5$ & Reference & - & Reference & - & Reference & - & Reference & - \\
\hline$>2.5$ & $1.562(1.185-2.058)$ & $0.002 *$ & $1.255(0.812-1.939)$ & 0.307 & $1.798(1.286-2.514)$ & $0.001^{*}$ & $1.419(0.851-2.364)$ & 0.180 \\
\hline LMR & & & & & & & & \\
\hline$\leq 2.9$ & Reference & - & Reference & - & Reference & - & Reference & - \\
\hline
\end{tabular}


Table 4 (continued)

\begin{tabular}{|c|c|c|c|c|c|c|c|c|}
\hline \multirow[t]{3}{*}{ Risk Factors } & \multicolumn{4}{|c|}{ PFS: cox regression analysis ( $N=266,227$ progression events) } & \multicolumn{4}{|c|}{ OS: cox regression analysis ( $N=248,161$ dead events) } \\
\hline & \multicolumn{2}{|l|}{ Univariable analysis } & \multicolumn{2}{|l|}{ Multivariate analysis } & \multicolumn{2}{|l|}{ Univariable analysis } & \multicolumn{2}{|l|}{ Multivariate analysis } \\
\hline & HR (95\% CI) & $P$ value & HR $(95 \% \mathrm{CI})$ & $P$ value & $\mathrm{HR}(95 \% \mathrm{CI})$ & $P$ value & $\mathrm{HR}(95 \% \mathrm{CI})$ & $P$ value \\
\hline$>2.9$ & $0.481(0.366-0.631)$ & $0.000 *$ & $0.478(0.279-0.820)$ & $0.007^{*}$ & $0.403(0.293-0.555)$ & $0.000 *$ & $0.524(0.279-0.985)$ & $0.045^{*}$ \\
\hline \multicolumn{9}{|l|}{ Treatment strategy } \\
\hline Chemotherapy & Reference & - & Reference & - & Reference & - & Reference & - \\
\hline $\begin{array}{l}\text { Chemotherapy + radi- } \\
\text { otherapy }\end{array}$ & $0.583(0.239-1.422)$ & 0.236 & $1.222(0.355-4.208)$ & 0.750 & $0.659(0.243-1.789)$ & 0.413 & $1.061(0.234-4.805)$ & 0.938 \\
\hline Surgery & $0.127(0.064-0.252)$ & $0.000 *$ & $0.471(0.197-1.125)$ & 0.090 & $0.236(0.119-0.468)$ & $0.000 *$ & $0.865(0.350-2.136)$ & 0.753 \\
\hline $\begin{array}{l}\text { Surgery }+ \\
\text { chemotherapy/ } \\
\text { radiotherapy }\end{array}$ & $0.338(0.227-0.506)$ & $0.000 *$ & $0.437(0.225-0.849)$ & $0.015^{*}$ & $0.366(0.224-0.597)$ & $0.000 *$ & $0.345(0.138-0.866)$ & $0.023^{*}$ \\
\hline Others & $0.808(0.458-1.425)$ & 0.462 & $0.746(0.363-1.537)$ & 0.427 & $1.140(0.597-2.177)$ & 0.692 & $1.094(0.498-2.402)$ & 0.824 \\
\hline Syn & $0.898(0.572-1.411)$ & 0.641 & & & $0.889(0.535-1.477)$ & 0.651 & & \\
\hline $\mathrm{CgA}$ & $0.883(0.655-1.190)$ & 0.413 & & & $0.824(0.580-1.170)$ & 0.279 & & \\
\hline CK18 & $2.182(0.809-5.890)$ & 0.123 & & & $2.220(0.701-7.034)$ & 0.175 & & \\
\hline TTF-1 & $1.126(0.744-1.704)$ & 0.576 & & & $1.065(0.667-1.700)$ & 0.792 & & \\
\hline Napsin A & $1.254(0.515-3.053)$ & 0.618 & & & $2.452(0.996-6.037)$ & 0.051 & & \\
\hline CK5/6 & $1.126(0.528-2.402)$ & 0.759 & & & $1.526(0.710-3.279)$ & 0.279 & & \\
\hline Ki67 & $1.016(1.009-1.024)$ & $0.000 *$ & $0.996(0.983-1.010)$ & 0.602 & $1.014(1.005-1.022)$ & $0.002 *$ & $0.997(0.983-1.012)$ & 0.732 \\
\hline P63 & $1.388(0.858-2.245)$ & 0.182 & & & $1.732(0.990-3.031)$ & 0.054 & & \\
\hline
\end{tabular}

PFS progression-free survival; OS overall survival; HR, hazard ratio; 95\% CI 95\% confidence intervals; BMI body mass index; SCLC smallcell lung cancer; $L C N E C$ large-cell neuroendocrine carcinoma; $A C$ atypical carcinoid; $T C$ typical carcinoid; $C E A$ carcinoembryonic antigen; $P L R$ platelet-lymphocyte ratio; NLR neutrophil-lymphocyte ratio; $L M R$ lymphocyte-monocyte ratio; Syn synaptophysin; $C g A$ chromogranin A; $C K 18$ Cytokeratin 18; TTF-1 thyroid transcription factor-1; CK5/6 Cytokeratin 5/6

${ }^{*} P<0.05$

95\% CI 1.196-2.526; $P=0.004 ; 5-7 \mathrm{~cm}: \mathrm{HR}, 1.750 ; 95 \%$ CI $1.115-2.747 ; P=0.015 ;>7 \mathrm{~cm}:$ HR $1.761 ; 95 \% \mathrm{CI}$ $1.041-2.980 ; P=0.035$ ), metastasis (lymph-node metastasis: HR 5.633; 95\% CI 2.963-10.707; $P=0.000$; distant metastasis: HR 2.761; 95\% CI 2.015-3.782; $P=0.000)$, pathotypes (taking SCLC as reference, LCNEC: HR 0.598 ; 95\% CI $0.313-1.144 ; P=0.120$; TC: HR 0.170 ; 95\% CI $0.054-0.538 ; P=0.003$; AC: HR $0.354 ; 95 \%$ CI $0.165-0.759 ; P=0.008$ ), Ki-67 index (HR 0.014; 95\% CI 1.005-1.022; $P=0.002)$, pretreatment CEA $(>5 \mathrm{ng} /$ ml: HR 2.147; 95\% CI 1.563-2.947; $P=0.000)$, PLR (> 152.5: HR 2.502; 95\% CI 1.820-3.441; $P=0.000$ ), NLR (>2.5: HR 1.798; 95\% CI 1.286-2.514; $P=0.001)$, LMR (> 2.9: HR $0.403 ; 95 \%$ CI $0.293-0.555 ; P=0.000$ ), and first-line treatment modalities (surgery: HR 0.236 ; 95\% CI $0.119-0.468 ; P=0.000$; surgery combined with chemotherapy or radiotherapy: HR, $0.36695 \% \mathrm{CI}, 0.224-0.597$; $P=0.000)$. In multivariate analysis, pretreatment CEA $(>5 \mathrm{ng} / \mathrm{ml}$ : HR $1.674 ; 95 \%$ CI $1.141-2.456 ; P=0.008)$ and LMR (> 2.9: HR 0.478; 95\% CI 0.279-0.820; $P=0.007$ ) were identified as independent prognostic factors for PFS. BMI (18.5-23.9 kg/m²: HR 0.249; 95\% CI 0.088-0.701; $P=0.009 ;>24 \mathrm{~kg} / \mathrm{m}^{2}:$ HR, $0.262 ; 95 \%$ CI $0.085-0.804$; $P=0.019$ ), lymph-node metastasis (HR $4.534 ; 95 \%$ CI
1.887-10.894; $P=0.001$ ), pathotypes (taking SCLC as reference, LCNEC: HR 3.154; 95\% CI 1.182-8.419; $P=0.022$; TC: HR $0.187 ; 95 \%$ CI $0.036-0.962$; $P=0.045)$, pretreatment PLR ( $>152.5$ : HR $2.305 ; 95 \%$ CI $1.311-4.055 ; P=0.004)$, and LMR ( $>2.9$ : HR $0.524 ; 95 \%$ CI $0.279-0.985 ; P=0.045)$ were identified as independent prognostic factors for OS. The Kaplan-Meier plots were used to generate survival curves (Figs. 2 and 3). The subgroups with pretreatment CEA $\leq 5 \mathrm{ng} / \mathrm{ml}, \mathrm{PLR} \leq 152.5$, NLR $\leq 2.5$, and LMR $>2.9$ presented a better PFS (Fig. 2) and OS (Fig. 3) (all $P<0.05$ ).

\section{Discussion}

In our retrospective study, patients presented distinctly different clinical characteristics among the pathotypes of PNETs. SCLC tends to occur among elderly individuals, most frequently with metastasis and have worse prognosis. With regard to immunohistochemical profile, SCLC had a higher positive rate of TTF-1. The Ki-67 index was significantly higher in SCLC and LCNEC than in carcinoid. Moreover, this current research indicated that smoking, pretreatment CEA $>5 \mathrm{ng} / \mathrm{ml}$, and poorly differentiated PNET 

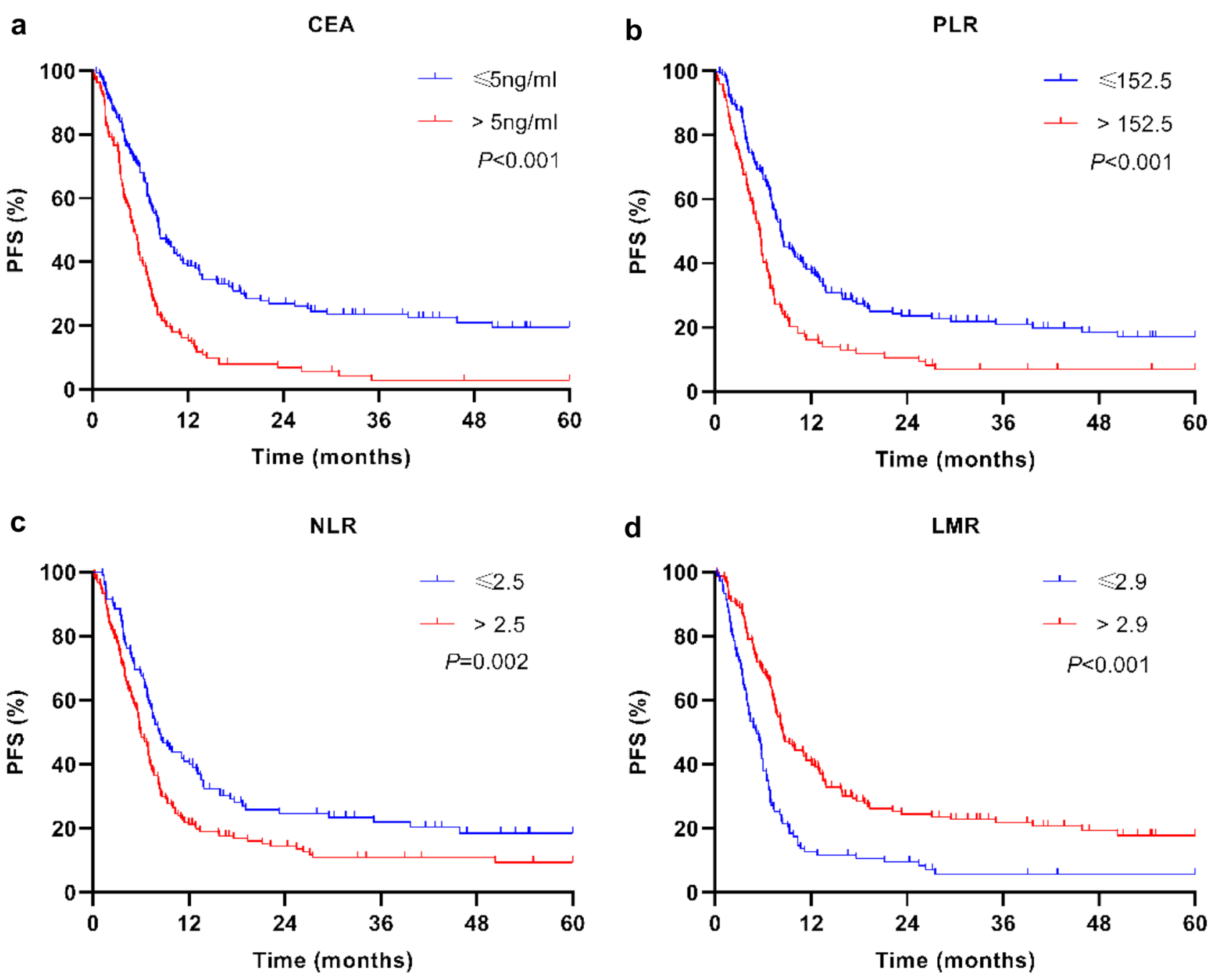

Fig. 2 PFS for the PNET patients in matched groups using KaplanMeier analysis and log-rank test. a PFS among PNET patients based on low pretreatment CEA $(\leq 5 \mathrm{ng} / \mathrm{ml})$ and high pretreatment CEA ( $>5 \mathrm{ng} / \mathrm{ml}$ ). b PFS among PNET patients based on low pretreatment PLR $(\leq 152.5)$ and high pretreatment PLR $(>152.5)$. c PFS among PNET patients based on low pretreatment NLR $(\leq 2.5)$ and high

pathotypes were independent risk factors for lymph-node metastasis. Smoking and pretreatment CEA $>5 \mathrm{ng} / \mathrm{ml}$ were independent risk factors for distant metastasis. Survival analysis showed that males, age at diagnosis $\geq 60$ years, $\mathrm{BMI}<18.5 \mathrm{~kg} / \mathrm{m}^{2}$, large tumor size, metastasis, poorly differentiated pathotypes, high Ki-67 index, low pretreatment LMR and increase of pretreatment CEA, PLR, and NLR were risk factors for OS. Surgery alone or surgery combined with chemotherapy and/or radiotherapy could prolong survival.

Previous studies have demonstrated the clinicopathological characteristics among subgroups of PNETs. Yeh and Chou (2014) and Kim et al. (2020) confirmed that age at diagnosis, gender, smoking, tumor size, metastasis, stage,

pretreatment NLR $(>2.5)$. d PFS among PNET patients based on low pretreatment LMR $(\leq 2.9)$ and high pretreatment $\operatorname{LMR}(>2.9)$. Abbreviations: PFS progression-free survival; PNET pulmonary neuroendocrine tumor; CEA carcinoembryonic antigen; PLR plateletlymphocyte ratio; NLR neutrophil-lymphocyte ratio; $L M R$ lymphocyte-monocyte ratio

and occurring disease progression were significantly different among SCLC, LCNEC, TC, and AC, consistent with our results.

Neuroendocrine marker expression also differed among subtypes of PNETs. TTF-1 was reported to be expressed by surfactant-producing type 2 pneumocytes and can also be detected in most small-cell and approximately $60-80 \%$ of lung adenocarcinomas (Bruno et al. 1995; Ordóñez 2000; Yatabe et al. 2002). Furthermore, the Ki-67 index represents the proliferation ability. There is a substantial amount of research, reporting that Ki-67 has great utility for SCLC/LCNEC from carcinoids, but typical and atypical carcinoids cannot be distinguished (Garg et al. 2019; Marchevsky et al. 2018; Naheed et al. 2019). In addition, 

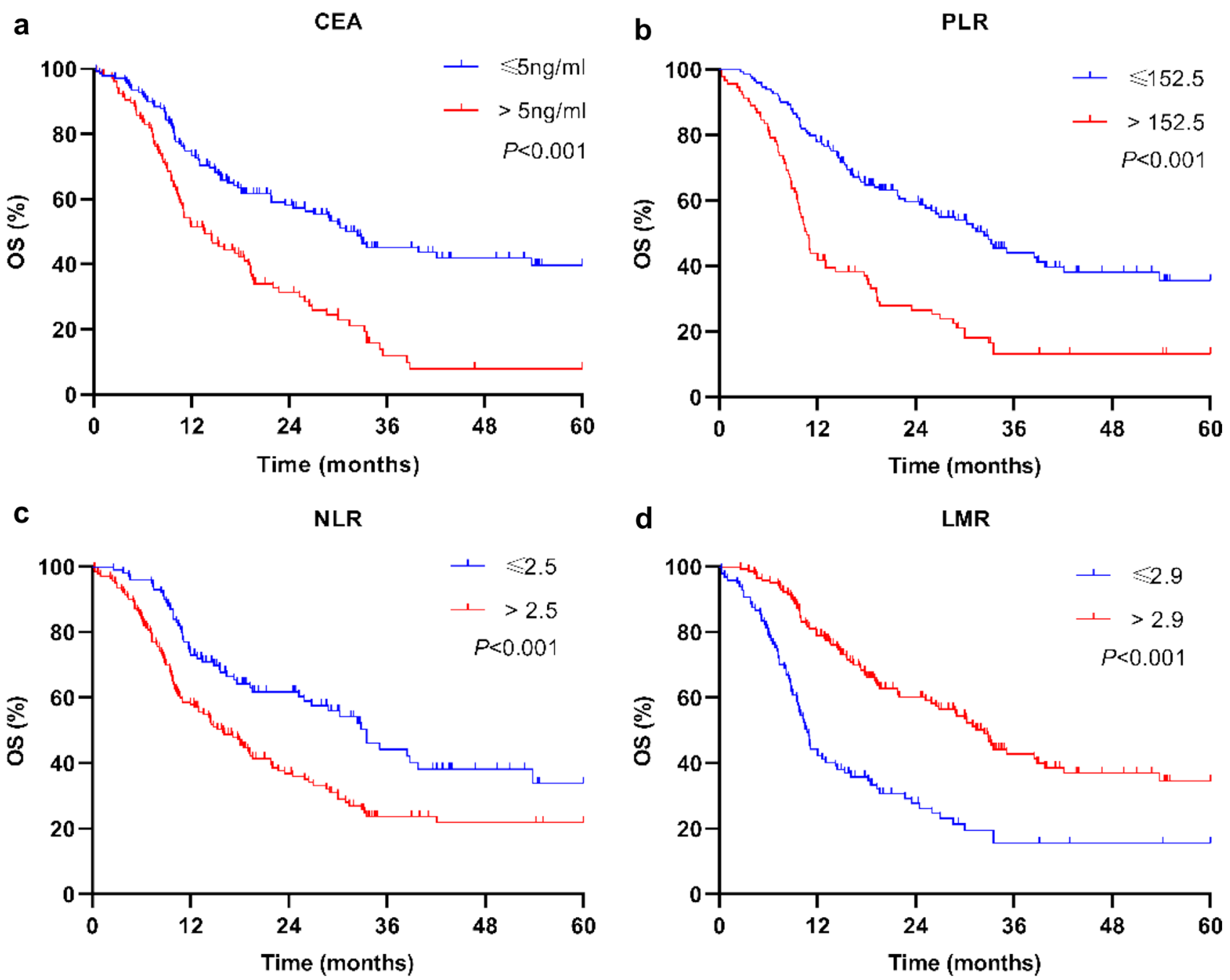

Fig. 3 OS for the PNET patients in matched groups using KaplanMeier analysis and log-rank test. a OS among PNET patients based on low pretreatment CEA $(\leq 5 \mathrm{ng} / \mathrm{ml})$ and high pretreatment CEA $(>5 \mathrm{ng} / \mathrm{ml}$ ). b OS among PNET patients based on low pretreatment PLR $(\leq 152.5)$ and high pretreatment PLR $(>152.5)$. c OS among PNET patients based on low pretreatment NLR $(\leq 2.5)$ and high

Thunnissen et al. (2017) suggested that cytokeratin CK8, CK18, CK7, and CK19 may be associated with SCLC. CK5/6, p63, and p40 are commonly used as immunohistochemical markers for squamous cell carcinoma of the lung (Matsukuma et al. 2018). However, there remains conflicting views on the immunohistochemical profile. The 2015 WHO classification of lung tumors suggested that Syn and $\mathrm{CgA}$ were recommended as first-hand choice for neuroendocrine markers (Travis et al. 2015), while Staaf et al. (2020) claimed that $\mathrm{CgA}$ had limited sensitivity. There are also different views on the prognostic role of immunohistochemical molecules. Hokari et al. (2020) found that highly expressed TTF-1 was associated with poor prognosis. The same results were obtained by Frost et al. (2020) later. In contrast, Dong

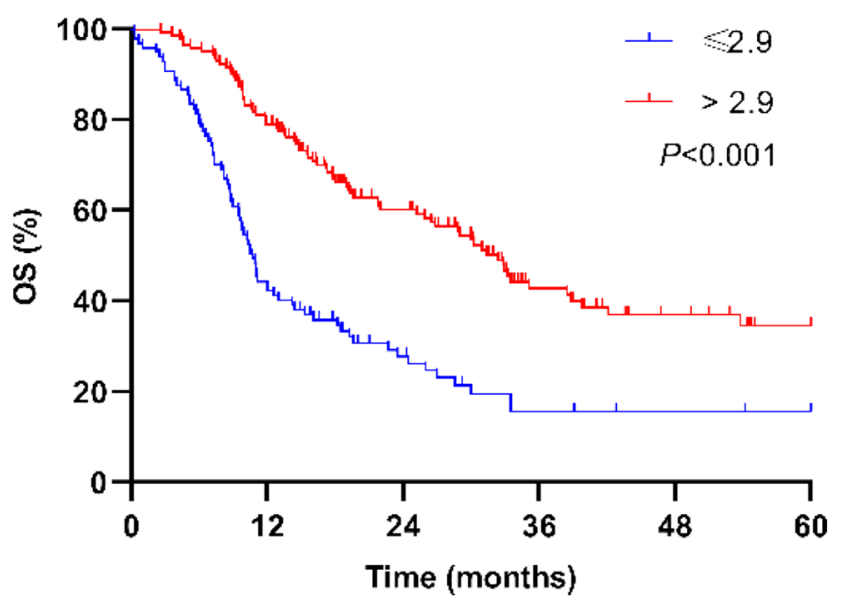

pretreatment NLR $(>2.5)$. d OS among PNET patients based on low pretreatment LMR $(\leq 2.9)$ and high pretreatment LMR $(>2.9)$. Abbreviations: $O S$ overall survival; PNET pulmonary neuroendocrine tumor; $C E A$ carcinoembryonic antigen; $P L R$ platelet-lymphocyte ratio; $N L R$ neutrophil-lymphocyte ratio; $L M R$ lymphocyte-monocyte ratio

et al. (2020) indicated that patients with positive expression of Napsin A had longer overall survival time than patients with negative expression, and the other NE markers were not associated with overall survival time. Our data showed a high positive rate of Syn, CgA, CK18, and TTF-1 in PNETs. In addition, the TTF-1 expression and Ki-67 index were significant different among the PNETs subtypes. The positivity of TTF-1 was much higher in SCLC, and the Ki-67 index was significantly higher in SCLC and LCNEC than in carcinoid tumors, which is consistent with the above reports. Besides, the Ki-67 index was proven to be of great significant in predicting PNET prognosis. Nonetheless, we did not find a prognostic role of other immunohistochemical molecules in PNETs. We suspect that the diverse result 
may be caused by the different study populations or methods used in research. Further research is needed to clarify the immunohistochemical molecular characteristics of PNETs.

Only pretreatment NLR showed significant differences among subgroups of PNETs at baseline, but PLR and LMR showed great prognostic value for PNETs. ROC analysis was performed to identify the optimal cut-off point of PLR, NLR, and LMR, and an optimal PLR, NLR, and LMR cutoff value of 152.5, 2.5, and 2.9, respectively. However, interracial and histological differences in the PLR, NLR, and LMR may influence the cut-off point (Okui et al. 2017). There are different reports on the PLR and NLR cut-off value. Shao and Cai (2015) defined the cut-off value of pretreatment PLR and NLR as 150 and 4.15, respectively. Okui et al. (2017) described the NLR cut-off value as 1.7, Suzuki et al. (2019) and Wang et al. (2020) held different views. The most appropriate cut-off value has not been established, and the current value could be viewed as arbitrary (Okui et al. 2017). Further researches are needed to determine the optimal cut-off value. In addition, pretreatment PLR, NLR, and LMR were first applied to all pathotypes of PNETs for prognostic analysis. Interestingly, we found that low pretreatment LMR, high pretreatment PLR, and NLR were associated with poor PFS and OS. These results were fairly comparable to the results of previous single pathology studies (Okui et al. 2017; Shao and Cai 2015; Suzuki et al. 2019; Wang et al. 2020).

There are several potential hypotheses regarding the mechanism by which low LMR, high NLR, and PLR promote cancer progression. Low LMR, high NLR, and PLR are equivalent to an increase in neutrophil count and a decrease in lymphocytes. Multiple studies have suggested that elevated neutrophil counts or lymphocytes loss may facilitate tumor development and progression by acting on tumor microenvironment. There are two major impacts toward tumors' progression. On one hand, inflammatory cells themselves play an important role in tumorigenesis and progression. Lymphocytes control many immune functions and lymphocyte loss owing to failed antitumor immunity (Lin and Pollard 2004). A high NLR indicates an imbalance in the immune response, which may impair normal antitumor functions, thus contributing to a worse prognosis for the host (Nagai et al. 2014; Xiao et al. 2013). On the other hand, inflammatory cells play a role in promoting tumor development by secreting a variety of inflammatory cytokines and chemokines, as well as proangiogenic factors (e.g., tumor necrosis factor, interleukin-1, interleukin-6, and vascular endothelial growth factor) (Balkwill and Mantovani 2001; Kusumanto et al. 2003). The exact mechanism of PLR, NLR, and LMR on tumor prognosis remains unclear yet. Further studies still need to be conducted to fully understand the molecular mechanism involved in these results. Inflammation within the tumor microenvironment can affect every aspect of tumor development and progression as well as response to therapy (Greten and Grivennikov 2019). In future research, inflammatory cells may be combined to guide the formulation of immunotherapy or chemotherapy.

For the analysis of the influence factors of survival, except for the above-mentioned immune-inflammation index, this current research showed that pretreatment CEA $>5 \mathrm{ng} / \mathrm{ml}$ was associated with metastasis, poor PFS, and OS in PNETs. Multiple studies have reported CEA as a tumor maker in the progression of lung cancer. (Grunnet and Sorensen 2012) pointed out that the serum level of CEA provided prognostic and predictive information on the risk of recurrence and death in NSCLC independent of treatment or study design. Kuo et al. (2020) implied that CEA was a prognostic factor associated with new metastasis, poor PFS, and OS in patients harboring epidermal growth factor receptor (EGFR) mutations. However, a few studies have reported the relationship between CEA and PNETs. Our study further confirmed the prognostic role of CEA. More attention should be given to tumor markers in clinical practice.

In terms of population inclusion, the number of LCNEC and carcinoid cases was relatively small. The immunohistochemical profile was incomplete, and the missing data for neuroendocrine marker expression may weaken the significant difference between groups. In addition, the baseline levels of the involved cases were not completely consistent (such as the age, stage, or first-line treatment modalities), and we did not discuss the guiding role of clinicopathological characteristics in treatment. Further research is needed to explore its guiding role in treatment.

\section{Conclusions}

PNETs are a group of highly heterogeneous tumors with different clinical manifestations, pathological features, and prognoses. Knowing the clinicopathological characteristics and immunophenotypes of PNETs is significant for diagnosis. Pretreatment PLR, LMR, and CEA have certain value in the prognosis of PNETs.

Acknowledgements This work was supported by the Nantong Science and Technology Project (MSZ19192)

Author contributions All authors participated in the data analyses, contributed to design of the work, data collection, analysis, and the writing of the manuscript, and approved the final version of the submitted manuscript. Co-authors are responsible for specific other parts of the work. In addition, authors have confidence in the integrity of the contributions of their co-authors. JF and SZ: designing, revising, supervising, and approval of the version to be published. JC: data collecting, analyzing, writing, and modifying of the manuscript. RZ: analyzing, revising, and editing. LX: designing, editing, revising, and supervising. YW: data collecting and analyzing. ZY: visiting by telephone and data collecting. XH: data collecting, writing, and revising. 
Funding This work was supported by the Nantong Science and Technology Project (MSZ19192).

Data availability The datasets generated and analyzed during the current study are available from the corresponding author on reasonable request.

\section{Declarations}

Conflict of interest The authors have no relevant financial or non-financial interests to disclose.

Ethical approval This retrospective study was approved by the ethics committee of Nantong University Affiliated Hospital and was conducted in accordance with the principles of the Declaration of Helsinki.

Consent to participate Informed consent was obtained from all individual participants included in the study.

Authorship clarified All authors agreed with the content, all gave explicit consent to submit, and we obtained consent from the responsible authorities at the institution where the work has been carried out, before the work is submitted.

Open Access This article is licensed under a Creative Commons Attribution 4.0 International License, which permits use, sharing, adaptation, distribution and reproduction in any medium or format, as long as you give appropriate credit to the original author(s) and the source, provide a link to the Creative Commons licence, and indicate if changes were made. The images or other third party material in this article are included in the article's Creative Commons licence, unless indicated otherwise in a credit line to the material. If material is not included in the article's Creative Commons licence and your intended use is not permitted by statutory regulation or exceeds the permitted use, you will need to obtain permission directly from the copyright holder. To view a copy of this licence, visit http://creativecommons.org/licenses/by/4.0/.

\section{References}

Anderson KL Jr, Mulvihill MS, Speicher PJ, Yerokun BA, Gulack BC, Nussbaum DP et al (2017) Adjuvant chemotherapy does not confer superior survival in patients with atypical carcinoid tumors. Ann Thorac Surg 104(4):1221-1230. https://doi.org/10.1016/j. athoracsur.2017.05.011

Balkwill F, Mantovani A (2001) Inflammation and cancer: back to virchow. Lancet 357(9255):539-545. https://doi.org/10.1016/ S0140-6736(00)04046-0

Bruno MD, Bohinski RJ, Huelsman KM, Whitsett JA, Korfhagen TR (1995) Lung cell-specific expression of the murine surfactant protein A (SP-A) gene is mediated by interactions between the SP-A promoter and thyroid transcription factor-1. J Biol Chem 270(12):6531-6536. https://doi.org/10.1074/jbc.270.12.6531

Chen Y, Wang W, Zhang X, Yu X, Xi K, Wen Y et al (2018) Prognostic significance of combined preoperative platelet-to-lymphocyte ratio and lymphocyte-to-monocyte ratio in patients undergoing surgery with stage IB non-small-cell lung cancer. Cancer Manag Res 10:5411-5422. https://doi.org/10.2147/CMAR.S177320

Daddi N, Schiavon M, Filosso PL, Cardillo G, Ambrogi MC, De Palma A et al (2014) Prognostic factors in a multicentre study of 247 atypical pulmonary carcinoids. Eur J Cardiothorac Surg 45(4):677-686. https://doi.org/10.1093/ejcts/ezt470
Dasari A, Shen C, Halperin D, Zhao B, Zhou S, Xu Y et al (2017) Trends in the incidence, prevalence, and survival outcomes in patients with neuroendocrine tumors in the United States. JAMA Oncol 3(10):1335-1342. https://doi.org/10.1001/jamaoncol.2017. 0589

Derks J, van Suylen RJ, Thunnissen E, den Bakker M, Groen H, Smit E et al (2017) Why we should improve current practice of diagnosing and treating pulmonary large cell neuroendocrine carcinomas in patients with advanced disease. Eur Respir J. https://doi.org/10. 1183/13993003.01658-2017

Derks JL, Leblay N, Thunnissen E, van Suylen RJ, den Bakker M, Groen H et al (2018) Molecular subtypes of pulmonary largecell neuroendocrine carcinoma predict chemotherapy treatment outcome. Clin Cancer Res 24(1):33-42. https://doi.org/10.1158/ 1078-0432.CCR-17-1921

Dingemans AC, Früh M, Ardizzoni A, Besse B, Faivre-Finn C, Hendriks LE et al (2021) Small-cell lung cancer: ESMO Clinical Practice Guidelines for diagnosis, treatment and follow-up. Ann Oncol 32(7):839-853. https://doi.org/10.1016/j.annonc.2021.03.207

Doll KM, Rademaker A, Sosa JA (2018) Practical guide to surgical data sets: surveillance, epidemiology, and end results (SEER) database. JAMA Surg 153(6):588-589. https://doi.org/10.1001/ jamasurg.2018.0501

Dong Y, Li Y, Liu R, Li Y, Zhang H, Liu H et al (2020) Secretagogin, a marker for neuroendocrine cells, is more sensitive and specific in large cell neuroendocrine carcinoma compared with the markers CD56, CgA Syn and Napsin A. Oncol Lett 19(3):2223-2230. https://doi.org/10.3892/ol.2020.11336

Frost N, Zhamurashvili T, von Laffert M, Klauschen F, Ruwwe-Glösenkamp C, Raspe M et al (2020) Pemetrexed-based chemotherapy is inferior to pemetrexed-free regimens in thyroid transcription factor 1 (TTF-1)-negative, EGFR/ALK-negative lung adenocarcinoma: a propensity score matched pairs analysis. Clin Lung Cancer 21(6):e607-e621. https://doi.org/10.1016/j.cllc.2020.05.014

García-Yuste M, Matilla JM, Cueto A, Paniagua JM, Ramos G, Cañizares MA et al (2007) Typical and atypical carcinoid tumours: analysis of the experience of the Spanish Multi-centric Study of Neuroendocrine Tumours of the Lung. Eur J Cardiothorac Surg 31(2):192-197. https://doi.org/10.1016/j.ejcts.2006.11.031

Garg R, Bal A, Das A, Singh N, Singh H (2019) Proliferation marker (Ki67) in sub-categorization of neuroendocrine tumours of the lung. Turk Patoloji Derg 35(1):15-21. https://doi.org/10.5146/ tjpath.2018.01436

Greten FR, Grivennikov SI (2019) Inflammation and cancer: triggers, mechanisms, and consequences. Immunity 51(1):27-41. https:// doi.org/10.1016/j.immuni.2019.06.025

Grunnet M, Sorensen JB (2012) Carcinoembryonic antigen (CEA) as tumor marker in lung cancer. Lung Cancer 76(2):138-143. https:// doi.org/10.1016/j.lungcan.2011.11.012

Gustafsson BI, Kidd M, Chan A, Malfertheiner MV, Modlin IM (2008) Bronchopulmonary neuroendocrine tumors. Cancer 113(1):5-21. https://doi.org/10.1002/cncr.23542

Hokari S, Tamura Y, Kaneda A, Katsura A, Morikawa M, Murai F et al (2020) Comparative analysis of TTF-1 binding DNA regions in small-cell lung cancer and non-small-cell lung cancer. Mol Oncol 14(2):277-293. https://doi.org/10.1002/1878-0261.12608

Kim M, Chung YS, Kim KA, Shim HS (2020) Genomic Profiling and clinicopathological characteristics of neuroendocrine tumors of the lung in east Asian patients. In Vivo 34(6):3375-3385. https:// doi.org/10.21873/invivo.12176

Kuo YS, Zheng MY, Huang MF, Miao CC, Yang LH, Huang TW et al (2020) Association of divergent carcinoembryonic antigen patterns and lung cancer progression. Sci Rep 10(1):2066. https:// doi.org/10.1038/s41598-020-59031-1

Kusumanto YH, Dam WA, Hospers GA, Meijer C, Mulder NH (2003) Platelets and granulocytes, in particular the neutrophils, form 
important compartments for circulating vascular endothelial growth factor. Angiogenesis 6(4):283-287. https://doi.org/10. 1023/B:AGEN.0000029415.62384.ba

Lin EY, Pollard JW (2004) Role of infiltrated leucocytes in tumour growth and spread. Br J Cancer 90(11):2053-2058. https://doi. org/10.1038/sj.bjc.6601705

Marchevsky AM, Hendifar A, Walts AE (2018) The use of Ki-67 labeling index to grade pulmonary well-differentiated neuroendocrine neoplasms: current best evidence. Mod Pathol 31(10):1523-1531. https://doi.org/10.1038/s41379-018-0076-9

Matsukuma S, Obara K, Utsumi Y, Miyai K, Takeo H, Oshika Y et al (2018) Focal positivity of immunohistochemical markers for pulmonary squamous cell carcinoma in primary pulmonary choriocarcinoma: a histopathological study. Oncol Lett 16(6):72567263. https://doi.org/10.3892/ol.2018.9525

Nagai S, Abouljoud MS, Kazimi M, Brown KA, Moonka D, Yoshida A (2014) Peritransplant lymphopenia is a novel prognostic factor in recurrence of hepatocellular carcinoma after liver transplantation. Transplantation 97(6):694-701. https://doi.org/10.1097/01. TP.0000437426.15890.1d

Naheed S, Holden C, Tanno L, Jaynes E, Cave J, Ottensmeier CH et al (2019) The utility of Ki-67 as a prognostic biomarker in pulmonary neuroendocrine tumours: protocol for a systematic review and meta-analysis. BMJ Open 9(8):e031531. https://doi.org/10. 1136/bmjopen-2019-031531

Nussbaum DP, Speicher PJ, Gulack BC, Hartwig MG, Onaitis MW et al (2015) Defining the role of adjuvant chemotherapy after lobectomy for typical bronchopulmonary carcinoid tumors. Ann Thorac Surg 99(2):428-434. https://doi.org/10.1016/j.athoracsur. 2014.08.030

Okui M, Yamamichi T, Asakawa A, Harada M, Saito M, Horio H (2017) Prognostic significance of neutrophil-lymphocyte ratios in large cell neuroendocrine carcinoma. Gen Thorac Cardiovasc Surg 65(11):633-639. https://doi.org/10.1007/s11748-017-0804-y

Ordóñez NG (2000) Value of thyroid transcription factor-1 immunostaining in distinguishing small cell lung carcinomas from other small cell carcinomas. Am J Surg Pathol 24(9):1217-1223. https://doi.org/10.1097/00000478-200009000-00004

Rekhtman N (2010) Neuroendocrine tumors of the lung: an update. Arch Pathol Lab Med 134(11):1628-1638. https://doi.org/10. 5858/2009-0583-RAR.1

Shao N, Cai Q (2015) High pretreatment neutrophil-lymphocyte ratio predicts recurrence and poor prognosis for combined small cell lung cancer. Clin Transl Oncol 17(10):772-778. https://doi.org/ 10.1007/s12094-015-1289-8

Staaf J, Tran L, Söderlund L, Nodin B, Jirström K, Vidarsdottir H et al (2020) Diagnostic value of insulinoma-associated protein 1 (INSM1) and comparison with established neuroendocrine markers in pulmonary cancers. Arch Pathol Lab Med 144(9):1075-1085. https://doi.org/10.5858/arpa.2019-0250-OA

Steuer CE, Behera M, Kim S, Chen Z, Saba NF, Pillai RN et al (2015) Atypical carcinoid tumor of the lung: a surveillance, epidemiology, and end results database analysis. J Thorac Oncol 10(3):479_ 485. https://doi.org/10.1097/JTO.0000000000000419

Suzuki R, Wei X, Allen PK, Cox JD, Komaki R, Lin SH (2019) Prognostic significance of total lymphocyte count, neutrophil-to-lymphocyte ratio, and platelet-to-lymphocyte ratio in limited-stage small-cell lung cancer. Clin Lung Cancer 20(2):117-123. https:// doi.org/10.1016/j.cllc.2018.11.013

Thunnissen E, Borczuk AC, Flieder DB, Witte B, Beasley MB, Chung JH et al (2017) The use of immunohistochemistry improves the diagnosis of small cell lung cancer and its differential diagnosis. An international reproducibility study in a demanding set of cases. J Thorac Oncol 12(2):334-346. https://doi.org/10.1016/j. jtho.2016.12.004

Travis WD, Brambilla E, Nicholson AG, Yatabe Y, Austin J, Beasley MB et al (2015) The 2015 World Health Organization classification of lung tumors: impact of genetic, clinical and radiologic advances since the 2004 classification. J Thorac Oncol 10(9):1243-1260. https://doi.org/10.1097/JTO.0000000000 000630

Wang H, Ding Y, Li N, Wu L, Gao Y, Xiao C et al (2020) Prognostic value of neutrophil-lymphocyte ratio, platelet-lymphocyte ratio, and combined neutrophil-lymphocyte ratio and platelet-lymphocyte ratio in stage IV advanced gastric cancer. Front Oncol 10:841. https://doi.org/10.3389/fonc.2020.00841

Xiao GQ, Liu C, Liu DL, Yang JY, Yan LN (2013) Neutrophil-lymphocyte ratio predicts the prognosis of patients with hepatocellular carcinoma after liver transplantation. World J Gastroenterol 19(45):8398-8407. https://doi.org/10.3748/wjg.v19.i45.8398

Yang Q, Xu Z, Chen X, Zheng L, Yu Y, Zhao X et al (2019) Clinicopathological characteristics and prognostic factors of pulmonary large cell neuroendocrine carcinoma: a large population-based analysis. Thorac Cancer 10(4):751-760. https://doi.org/10.1111/ 1759-7714.12993

Yatabe Y, Mitsudomi T, Takahashi T (2002) TTF-1 expression in pulmonary adenocarcinomas. Am J Surg Pathol 26(6):767-773. https://doi.org/10.1097/00000478-200206000-00010

Yeh YC, Chou TY (2014) Pulmonary neuroendocrine tumors: study of 90 cases focusing on clinicopathological characteristics, immunophenotype, preoperative biopsy, and frozen section diagnoses. J Surg Oncol 109(3):280-286. https://doi.org/10.1002/jso.23497

Publisher's Note Springer Nature remains neutral with regard to jurisdictional claims in published maps and institutional affiliations. 